\title{
A non-resonant fiber scanner based on an electrothermally-actuated MEMS stage
}

\author{
Xiaoyang Zhang ${ }^{1}$, Can Duan ${ }^{1}$, Lin Liu ${ }^{1}$, Xingde $\mathrm{Li}^{2}$ and Huikai Xie ${ }^{1}$ \\ ${ }^{1}$ Dept. of Electrical \& Computer Engineering, University of Florida, Gainesville, FL 32611 \\ ${ }^{2}$ Dept. of Biomedical Engineering, Johns Hopkins University, Baltimore, MD 21205 \\ E-mail: xzhang292@ufl.edu Tel: +1-352-281-3355
}

\begin{abstract}
Scanning fiber tips provides the most convenient way for forward-viewing fiber-optic microendoscopy. In this paper, a distal fiber scanning method based on a large-displacement MEMS actuator is presented. A single-mode fiber is glued on the micro-platform of an electrothermal MEMS stage to realize large range non-resonantscanning. The micro-platform has a large piston scan range of up to $800 \mu \mathrm{m}$ at only $6 \mathrm{~V}$. The tip deflection of the fiber can be further amplified by placing the MEMS stage at a proper location along the fiber. A quasi-static model of the fiber-MEMS assembly has been developed and validated experimentally. The frequency response has also been studied and measured. A fiber tip deflection of up to $1650 \mu \mathrm{m}$ for the 45 mm-long movable fiber portion has been achieved when the MEMS electrothermal stage was placed $25 \mathrm{~mm}$ away from the free end. The electrothermally-actuated MEMS stage shows a great potential for forward viewing fiber scanning and optical applications.
\end{abstract}

Keywords: Distal fiber scanning, fiber scanner, electrothermal actuation, microactuator, MEMS stage, micro-platform, large range

\section{Introduction}

Due to their low optical loss, small diameter and mechanical flexibility, optical fibers have been widely used for light delivery and signal collection in numerous endoscopic optical three-dimensional (3D) imaging systems such as endoscopic optical coherence tomography (OCT), endoscopic nonlinear optical microscopy and endoscopic confocal microscopy for internal organs imaging where the space is very limited [1-9]. Such optical 3D imaging techniques can obtain high-resolution, cross-sectional structural information of tissue samples, but require lateral or transverse scanning of the light beam to acquire 3D images. Various scanning mechanisms have been employed at either the proximal or distal end of the fiber [3-9]. Proximal scanning typically uses fiber bundles, which is easy to realize high-degree miniaturization [3], but the resolution is limited by the diameter of individual fibers and the spacing between fibers. Distal scanning involves only a single fiber. There are two distal fiber scanning types. One is to scan the light beam emitting out of the distal end of the fiber. This optical scanning is done in free space either by a rotating prism powered by a micromotor [4] or a scanning MEMS micromirror [5]. Using MEMS micromirrors or micromotors can easily realize side-view scanning, but it is challenging to generate front-view scanning as at least two mirrors are needed to change the direction of the light beam which may bring difficulties for assembling and further miniaturization. The other distal scanning type is to directly scan the fiber tip at its distal end, which is similar way with the fiber-MEMS assembly reported in this paper. Several methods have been reported, such as piezoelectric tube actuators [6][7], electrostatically driven actuators [8], and 
electromagnetically excited actuators [9]. Although this type requires large actuation force, directly scanning distal fiber tips can easily realize forward-view scanning and has great potential to minimize the scanning probe size.

Among these actuation methods of fiber scanners, electrostatic and piezoelectric actuators typically require resonant scanning due to limited force or displacement range. So, their scan patterns are Lissajous or spiral [6][8], leading to non-uniform spatial coverage. A non-resonant piezo bimorph actuator-based fiber scanner was reported, but the operation voltage was over 200V [7]. A non-resonant electromagnetic actuator was also reported to scan a fiber tip [9], but the obtained field of view was only $69 \mu \mathrm{m}$. Electrothermal bimorph actuator based micromirrors have been demonstrated to achieve large scan range at low drive voltage [10][11]. With the advantages in actuation range and force [12][13], electrothermal actuation may have the potential for scanning fiber tips at non-resonant mode.

In this paper we present a fiber scanner that utilizes an electrothermal MEMS stage to drive a bare single-mode fiber that can operate at both non-resonant and resonant mode. In section 2, the concept of the scanning system using MEMS stage is introduced. Theoretical modeling of the quasi-static performance and resonance modes are studied in section 3. FEM simulation results using COMSOL are also presented in contrast with the theoretical modeling and experiments results. In section 4, the characterization of the MEMS stage and fiber-stage scanning system are reported. For a $45 \mathrm{~mm}$ long single-mode fiber, a large static scan range up to $1600 \mu \mathrm{m}$ has been achieved with the MEMS stage placed at $25 \mathrm{~mm}$ away from the fiber tip. The maximum operation voltage for the electrothermally-actuated MEMS stage is no more than $6 \mathrm{~V}$ at non-resonant mode.

\section{Working principle}

\subsection{Concept of the distal fiber system}

A 3-D schematic of the proposed distal fiber system is shown in Fig. 1, which consists of a MEMS stage and a bare single-mode fiber. The fiber is fixed with a movable tip. The MEMS stage, as shown in the inset in Fig. 1, is composed of a micro-platform, a group of bimorph actuators and a substrate. The micro-platform of the MEMS stage is attached to an intermediate point of the fiber tip, and thus divides the movable fiber portion into two parts: a fixed end and a free end. When the micro-platform is actuated by the bimorph actuators, the mechanical compliance of the fiber fixed end allows it to be moved up and down. The actuation force needed mainly depends on the stiffness of the fixed end. The tip of the movable fiber portion will have a much larger displacement than the micro-platform due to angular amplification.

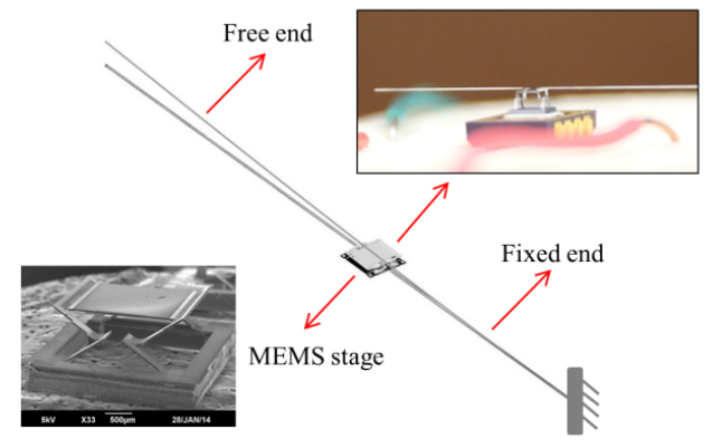

Fig. 1 Schematic of the distal fiber-MEMS stage scanning system. 


\subsection{Working principle of the MEMS stage}

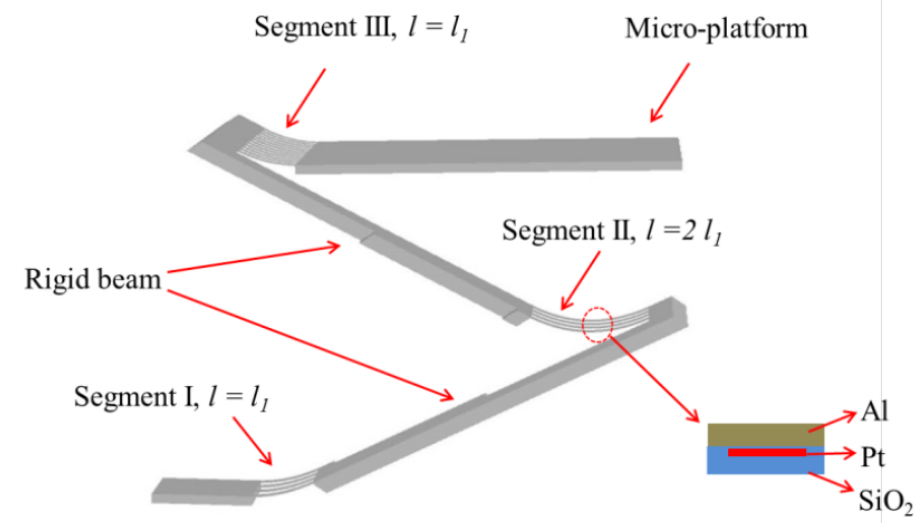

Fig. 2 Cross-sectional view schematics of three-segment bimorph LVD actuator design with no lateral shift.

The MEMS stage takes advantage of the large-vertical-displacement (LVD) bimorph actuator design which provides both moderate actuation force and large moveable range. The LVD bimorph actuator design with no lateral shift has been reported in [10]. The MEMS stage has two LVD bimorph actuators holding a silicon based micro-platform. $\mathrm{Al}$ and $\mathrm{SiO}_{2}$ are chosen as the top and bottom layers of the bimorph, respectively, because of their large difference in coefficient of thermal expansion (CTE). As shown in Fig. 2, three bimorph segments (I, II, III) are employed to cancel the lateral shift and generate large vertical displacement. The radii of curvature of the three bimorph segments are the same as they have the same layer structure. Thus, if the lengths of segments I and III are the same and equal to half of the length of segment II, then the in-plane shifts from segments I and segment III are canceled by segment II. At the same time, the vertical displacements of all three segments will be added up to produce a large pure out-of-plane displacement. The rigid segments with relatively thick Si layer underneath are included to increase the displacement range. An embedded $\mathrm{Pt}$ heater [19] is employed to generate temperature change across the actuator. The micro-platform of the MEMS stage will stay above the substrate due to the residual stresses in the bimorph after structure release. When an electrical voltage is applied, the bimorph temperature will increase and the micro-platform will move down toward the substrate because the CTE of Al is greater than that of $\mathrm{SiO}_{2}$. The SEMs of the whole device and close-view of the bimorph actuator are shown in Fig. 3. Two sets of LVD bimorph actuators are placed on the opposite sides of the micro-platform. When the same voltages are applied to both sets of actuators simultaneously, the micro-platform will perform a pure out-of-plane motion, which will move the fiber vertically in the system as shown in Fig. 1. 


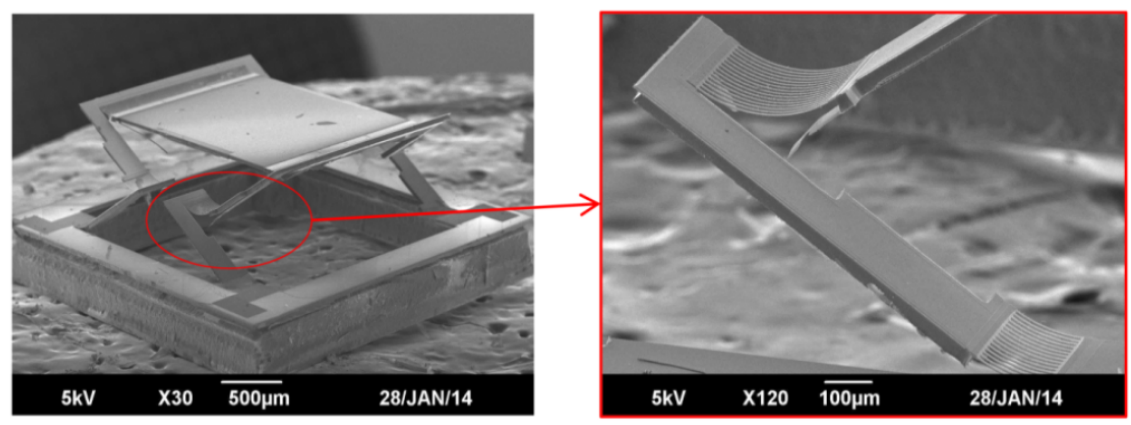

Fig. 3 SEMs of the MEMS stage and close-view of the LVD bimorph actuator.

3. Modeling

3.1 Quasi-static analysis

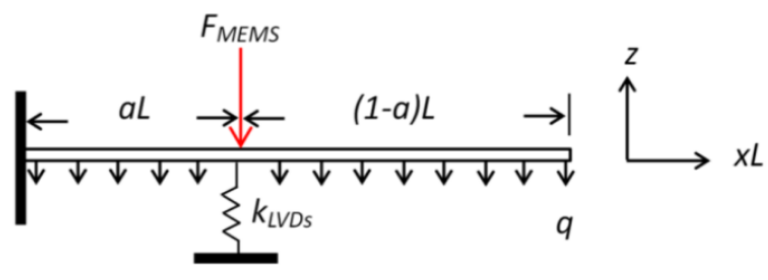

Fig. 4 Illustration of the force acting on the fiber.

The fiber-MEMS assembly shown in Fig.1 can be simplified as a model shown in Fig.4. Since the contact between the fiber and the MEMS stage is small and the tip deflection is very small compared with the length of the fiber, the fiber-to-MEMS contact is modeled as a point contact. For the fiber, the actuation force from the MEMS stage is a point force, which comes from the electrothermal force and is induced by the temperature change of the bimorph actuators of the MEMS stage [15]. The mass of the fiber can be considered as a distributed force along the fiber length, as shown in Fig. 4. The relation between the displacement of the micro-platform, $\delta_{s}$, and the corresponding displacement at the fiber tip, $\delta_{\text {tip }}$, is given by [16],

$$
\delta_{\text {tip }}=\delta_{s}\left[1+\frac{3(1-a)}{2 a}\right]+\frac{q g L^{4}}{8 E I}
$$

where $L$ is the total length of the movable fiber portion, $a$ is the length ratio of the fixed end, $E$ is the Young's modulus of the fiber, $I$ is the moment of inertia of the fiber, $q$ is the mass per unit fiber length and $g$ is the acceleration of gravity. The second term of Eq. (1) is constant for a given fiber length and it is the tip deflection caused by the weight of the fiber. For a typical fiber length $L=30$ $\mathrm{mm}$, this tip deflection is about $27 \mu \mathrm{m}$, which is negligible compared to the millimeter deflection range generated by the MEMS stage. $\left[1+\frac{3(1-a)}{2 a}\right]$ is the amplification factor between the tip deflection and the displacement of the micro-platform.

The MEMS-fiber assembly must be considered as a multi-degree-of-freedom system since -the fiber tip has both displacements in z-axis and tilt angle. Here we focus on the fiber-MEMS contact point, where the tilt angle is not considered due to the small platform size compared with the length 
of fiber. The MEMS LVD bimorph actuators and the fiber fixed end have comparable stiffness, So for convenience, energy method is used to do the force analysis. At steady state, the input electrical energy will be converted into three parts: (1) the thermal energy due to the temperature increase along the LVD bimorphs caused by Joule heating; (2) the elastic potential energy stored in the spring-like LVD bimorph actuators and in the cantilever-like fiber fixed end, which is determined by the displacement of the micro-platform; and (3) the thermal dissipation to the substrate and ambiance. Here the gravitational potential energies of the micro-platform and the movable fiber portion are negligible due to their so small mass.

For the LVD MEMS mirror shown in Fig. 3, the thermal isolation between the LVD actuators and the micro-platform are pure $\mathrm{SiO}_{2}$ bridges, the thermal resistance is about $1.2 \times 10^{4} \mathrm{~K} / \mathrm{W}$ and much greater than the equivalent thermal resistance of LVD bimorph actuator, about $900 \mathrm{~K} / \mathrm{W}$, which is determined by the thermal resistance from the bimorph actuator to substrate and the convection loss [14][24]. So less than 7\% of the thermal power will flow into the micro-platform and attached fiber. Thus, at the same applied voltage, the temperature changes on the LVD bimorphs are proximately equal with and without the fiber attached to the micro-platform. This is true for the thermal dissipation, too. Therefore, with a fiber loaded on the micro-platform, part of the elastic energy stored in the LVD bimorphs is transferred to the fiber, i.e.,

$$
\frac{k_{L V D s} \delta_{p}{ }^{2}}{2}=\frac{k_{L V D s} \delta_{s}{ }^{2}}{2}+\frac{k_{f} \delta_{s}{ }^{2}}{2}
$$

where $k_{L V D s}$ and $k_{f}$ are the effective stiffness of the LVD bimorphs and the fiber fixed end, respectively, and $\delta_{p}$ and $\delta_{s}$ are the micro-platform displacements without and with fiber attached, respectively.

For the LVD bimorph actuator design, the displacement with no loading is already studied and given by [17],

$$
\begin{gathered}
\delta_{p}=2 L_{r}\left[\sin \theta_{0}-\sin \left(\theta_{0}-\theta_{t}\right)\right] \\
\theta_{t}=\beta \frac{l_{b}}{t_{b}} \Delta \alpha \Delta T
\end{gathered}
$$

Here $L_{r}$ is the length of the rigid beam, $\theta_{0}$ is the initial curved angle due to residual stress, $\theta_{t}$ is the thermally induced arc angle of bimorph segment $\mathrm{I}$, and $l_{b}$ and $t_{b}$ are respectively the length and thickness of bimorph segment I. $\beta$ is a constant determined by material properties and layer thicknesses of the bimorph [18], and the value of $\beta$ for the $\mathrm{Al}_{-} \mathrm{SiO}_{2}$ bimorph design in this work is 1.5. $\Delta \alpha$ is the CTE difference of the two bimorph materials, and $\Delta T$ is the average temperature change of the LVD bimorph.

An electro- thermo-mechanical lumped element model (LEM) of the bimorph actuator has been developed by Todd and Xie [19], from which the average temperature change of the bimorph is determined by the applied voltage, $\mathrm{V}$, across the Pt heater, i.e.,

$$
\Delta T(V)=\frac{1}{2 \xi}\left(\sqrt{\frac{4 \xi R_{T}}{R_{E 0}} V^{2}+1}-1\right)
$$

where $\xi$ is the temperature coefficient of the Pt heater resistance, $\mathrm{R}_{\mathrm{T}}$ is the equivalent average thermal resistance of the bimorph, and $\mathrm{R}_{\mathrm{E} 0}$ is the initial electrical resistance of the Pt heater at the reference temperature. From Eq. (1) and (2) (5), the fiber tip deflection can be given by

$$
\delta_{\text {tip }}=\left[1+\frac{3(1-a)}{2 a}\right] \times \sqrt{\frac{k_{L V D S}}{k_{L V D S}+k_{f}}} \delta_{p}
$$




$$
=\left[1+\frac{3(1-a)}{2 a}\right] \sqrt{\frac{k_{L V D S}}{k_{L V D S}+k_{f}}} 2 L_{r}\left\{\sin \theta_{0}-\sin \left[\theta_{0}-\beta \frac{l_{b}}{t_{b}} \Delta \alpha \frac{1}{2 \xi}\left(\sqrt{\frac{4 \xi R_{T}}{R_{E 0}} V^{2}+1}-1\right)\right]\right\}
$$

The structure and material parameters of the LVD actuator are shown in Table 1. The thermal resistance is mainly the conduction from the LVD bimorphs to the substrate and the convection from the bimorph surfaces. The average heat loss coefficient on $\mathrm{Al}-\mathrm{SiO}_{2}$ bimorphs was found to be 188 $\mathrm{W} / \mathrm{m}^{2} / \mathrm{K}$, accounting for heat loss by thermal diffusion and convection through air [20]. Thus the thermal resistance of the LVD bimorph actuators is calculated to be about $900 \mathrm{~K} / \mathrm{W}$.

Table 1. Structure and material parameters of the LVD actuator and fiber

\begin{tabular}{lcc}
\hline Parameters & Symbol & Value \\
\hline Length of LVD actuator segment I & $l_{b}$ & $150 \mu \mathrm{m}$ \\
Thickness of LVD actuator segment I & $t_{b}$ & $2.2 \mu \mathrm{m}$ \\
Length of LVD actuator rigid beam & $L_{r}$ & $860 \mu \mathrm{m}$ \\
Equivalent stiffness of the LVDs & $k_{L V D s}$ & $0.3 \mathrm{~N} / \mathrm{m}$ \\
Initial curvature of LVD actuator segment I & $\theta_{0}$ & $0.7 \mathrm{rad}$ \\
Layer thickness related constant & $\beta$ & 1.5 \\
Resistance of the Pt heater at $25^{\circ} \mathrm{C}$ & $\mathrm{R}_{\mathrm{E} 0}$ & $85 \Omega$ \\
Pt heater TCR & $\xi$ & $0.003 \mathrm{~K}^{-1}$ \\
CTE difference between Aluminum and $\mathrm{SiO}_{2}$ & $\Delta \alpha$ & $22.7 \times 10^{-6} \mathrm{~K}^{-1}$ \\
Equivalent average thermal resistance of the bimorph & $\mathrm{R}_{\mathrm{T}}$ & $900 \mathrm{~K} / \mathrm{W}$ \\
\hline
\end{tabular}

The tip deflection is determined by both the stiffness of the fixed end and the amplification factor. For a given total fiber length, the displacement of the fiber-MEMS contact point decreases with the fixed end length ratio $a$, but the amplification factor increases with $a$. Thus, there is an optimal ratio for maximum tip deflection, as shown in Fig. 5(a). Fig. 5(b) shows the relation between the tip deflection and the input voltage to the MEMS stage for a $45 \mathrm{~mm}$-long fiber with different fixed end length ratios. From the figure we can clearly see that a quasi-static range greater than $1.5 \mathrm{~mm}$ can be achieved with this electrothermally actuated fiber scanning at only $6 \mathrm{~V}$.

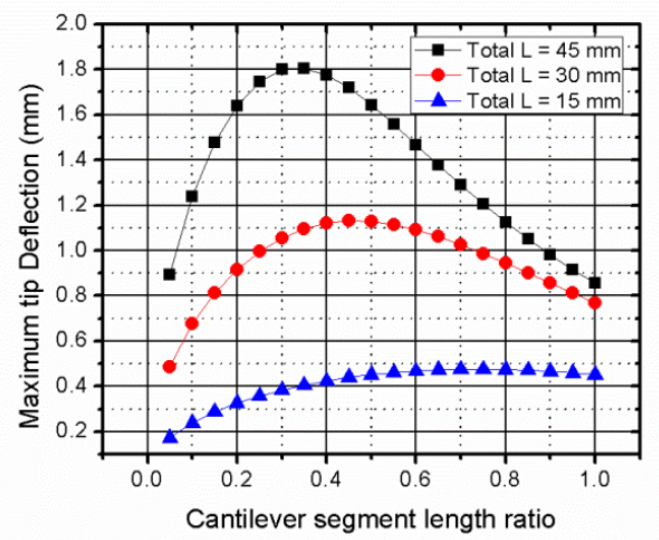

(a) 


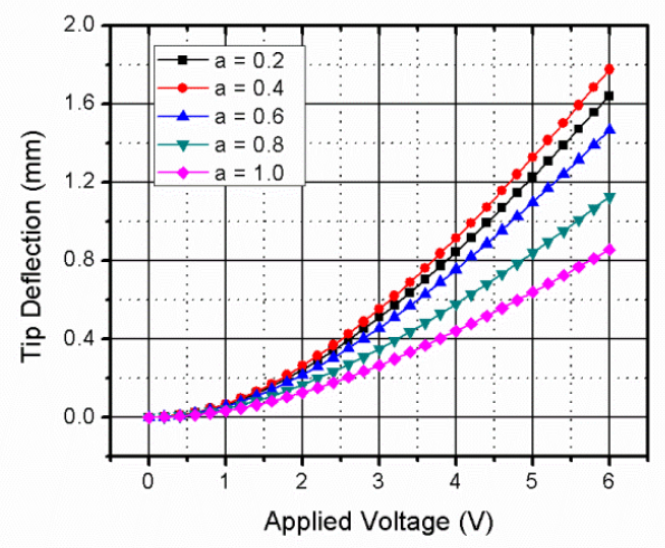

(b)

Fig. 5 (a) Maximum tip deflection for fiber with different total lengths and ratios. (b) Tip deflection verus applied voltage for a $45 \mathrm{~mm}$ long fiber for fixed end length ratio $\mathrm{a}=0.2,0.4,0.6,0.8,1$.

\subsection{Fundamental Resonance}

The fiber-MEMS assembly can work at both non-resonant and resonant modes. Its fundamental resonant mode is analyzed here, which may be adapted for various optical scanning systems. The fiber-MEMS assembly can be modeled as an overhanging cantilever beam with an intermediate elastic support. The frequency response of this system is mainly determined by three parameters: the location and stiffness of the elastic support and the total length of the fiber cantilever [21]. In our case, the MEMS stage acts as the intermediate elastic support whose stiffness can be considered constant under certain voltage bias range. The location of the elastic support determines the lengths of both the fixed end and free end of the fiber.

As shown in Fig. 4, the fiber is a uniform cantilever beam. Then the frequency behavior of the fiber-MEMS stage system is governed by classical Bernoulli-Euler theory. The fundamental modal equation for the vibration of the fiber is readily given by [22]:

$$
\begin{gathered}
\frac{\partial^{4} z(x)}{\partial^{4} x}-\lambda^{4} z(x)=0 \\
\lambda^{4}=\frac{\omega^{2} q L^{4}}{E I}
\end{gathered}
$$

where $z(x)$ is the transverse displacement of the fiber at normalized position $\mathrm{x}$, and $\omega$ is the angular frequency of a vibration mode of the overhanging cantilever-spring system. The parameters in Eq. (8) are the same as those shown in Fig. 4. The general solution to Eq. (7) is separated into two parts which have different boundary conditions. For the fixed fiber end, we have

$$
z_{1}(x)=C_{1}[\sinh \lambda x-\sin \lambda x]+C_{2}[\cosh \lambda x-\cos \lambda x], 0 \leq x \leq a
$$

For the free fiber end, the transverse displacement is given by

$$
\begin{aligned}
z_{2}(x)= & C_{3}[\sinh \lambda(x-1)+\sin \lambda(x-1)] \\
& +C_{4}[\cosh \lambda(x-1)+\cos \lambda(x-1)], a \leq x \leq 1
\end{aligned}
$$

The continuity conditions of the displacement, slope and bending moment at the support position yield:

$$
\begin{aligned}
& z_{1}(a)=z_{2}(a) \\
& z_{1}^{\prime}(a)=z_{2}^{\prime}(a)
\end{aligned}
$$




$$
z_{1}^{\prime \prime}(a)=z_{2}^{\prime \prime}(a)
$$

The continuity of the shear force at the support point, which is related to the LVDs' effective spring constant [22], yields:

$$
\frac{k_{L V D S} L^{3}}{E I}=\frac{z_{1}^{\prime \prime \prime}(a)-z_{2}^{\prime \prime \prime}(a)}{z_{1}(a)}
$$

Applying Eqs. (9) and (10) to Eqs. (11) (14), we obtain a series of equations with four unknowns $C_{1}, C_{2}, C_{3}$, and $C_{4}$. To guarantee non-trivial solutions, we must have

$$
\operatorname{det}(\mathrm{A})=0
$$

$$
A=\left|\begin{array}{cccc}
\sinh \lambda a-\sin \lambda a & \cosh \lambda a-\cos \lambda a & -\sinh \lambda(a-1)-\sin \lambda(a-1) & -\cosh \lambda(a-1)-\cos \lambda(a-1) \\
\cosh \lambda a-\cos \lambda a & \sinh \lambda a+\sin \lambda a & -\cosh \lambda(a-1)-\cos \lambda(a-1) & -\sinh \lambda(a-1)-\sin \lambda(a-1) \\
\sinh \lambda a+\sin \lambda a & \cosh \lambda a-\cos \lambda a & -\sinh \lambda(a-1)+\sin \lambda(a-1) & -\cosh \lambda(a-1)+\cos \lambda(a-1) \\
(\cosh \lambda a-\cos \lambda a)-\frac{k_{L V D L} L^{3}}{E I \lambda^{3}}(\sinh \lambda a-\sin \lambda a) & (\sinh \lambda a-\sin \lambda a)-\frac{k_{L V D S} L^{3}}{E I \lambda^{3}}(\cosh \lambda a-\cos \lambda a) & -\cosh \lambda(a-1)+\cos \lambda(a-1) & -\sinh \lambda(a-1)-\sin \lambda(a-1)
\end{array}\right|
$$

Solving Eq. (15) for the smallest non-zero solution of $\lambda$, and then the fundamental resonance frequency of the fiber-stage system can be calculated from Eq. (8) and is plotted in Fig. 6. As shown in Fig. 6(a), the fundamental resonance frequency increases with the fixed end length ratio moderately, while shortening the total fiber length can increase the resonance dramatically. Matlab calculation on Eq. (15) is also compared to COMSOL simulation. As shown in Fig. 6(b), the analytical modeling agrees with the FEM modeling and the error is less than 15\%. Fig. 6(c) shows the mode shape of a fundamental resonance, which is the vertical vibration of the movable fiber portion, as well as shown in Fig. 10(b).

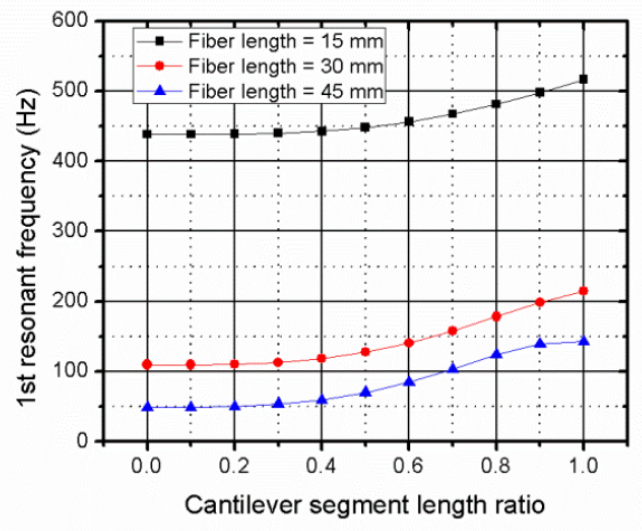

(a)

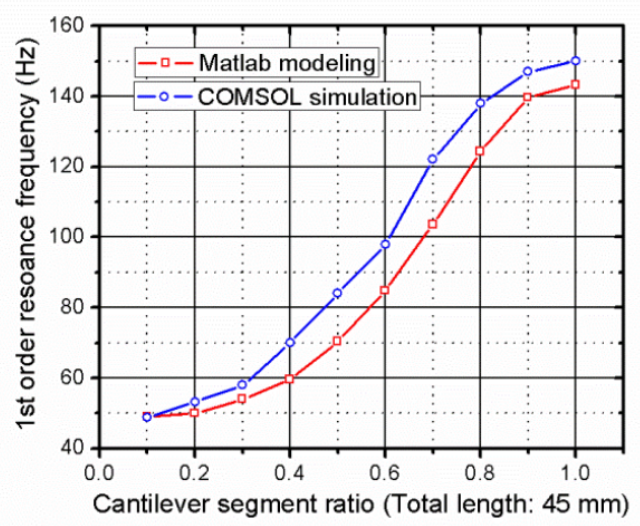

(b) 


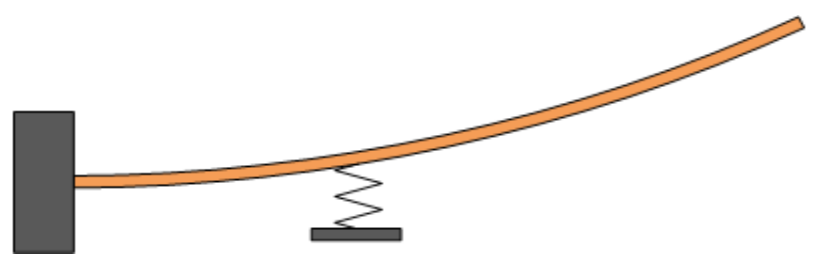

(c)

Fig. 6 (a) The fundamental resonance frequency for $15 \mathrm{~mm}, 30 \mathrm{~mm}, 45 \mathrm{~mm}$ long fiber with different fixed end length ratios. (b) Matlab and COMSOL simulation results: fundamental resonance frequency versus fixed end length ratio for $45 \mathrm{~mm}$ long fiber. (c) Mode shape of the fundamental resonance.

\section{Experiment results and analysis}

\subsection{Characterization of the MEMS stage.}

When two pairs of LVDs are actuated simultaneously with same inputs, the micro-platform moves down. The piston displacement of the micro-platform under a range of applied voltage was measured using an Olympus BX51 microscope and a QC200 geometry measuring system. The measurement error was $\pm 0.5 \mu \mathrm{m}$. The frequency response of the MEMS stage was measured using a Doppler Laser Vibrometer. Fig. 7(a) shows the vertical displacement versus voltage and the frequency responses under different voltage bias. The low frequency response is dominated by thermal domain which shows a dramatic magnitude drop due to the low thermal cut-off frequency [14]. Note that, as shown in Fig. 7(b), the resonant frequency of the first mode shifts when the bias voltage changes: higher bias voltage leads to lower resonant frequency. This effect is mainly caused by the Young's modulus temperature dependence of the bimorph materials [23] and the stiffness change due to the bimorph shape change.

The vertical displacements at various voltages are plotted in Fig. 7(a), both experiment results and modeling result from Eq. (3). The stiffness of the MEMS stage can be calculated from $k_{L V D S}=$ $m_{p} \times(2 \pi f)^{2}$, where $m_{p}$ is the mass of the micro-platform and $\mathrm{f}$ is the resonant frequency plotted in Fig. $7(\mathrm{~b}), k_{L V D S}$ ranges from $0.26 \mathrm{~N} / \mathrm{m}$ to $0.32 \mathrm{~N} / \mathrm{m}$ at different biases. Thus, the maximum electrothermal force the MEMS stage can provided is about $200 \mu \mathrm{N}$.

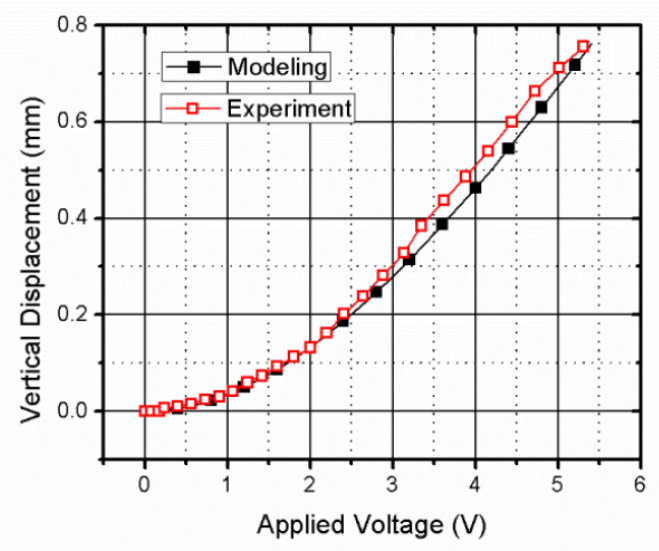

(a) 


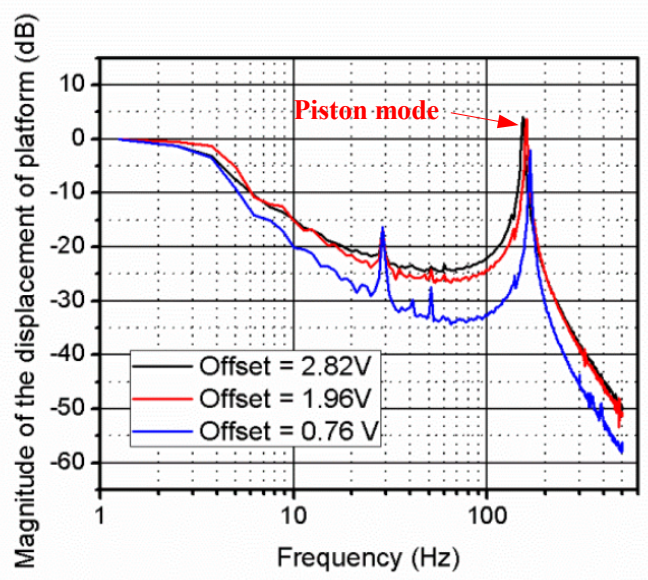

(b)

Fig. 7 (a) Vertical displacement versus applied voltage without fiber attached. (b) Frequency responses with different voltage bias for the MEMS stage without fiber attached.

\subsection{Experiment results with fiber attached}

A free-space experiment setup is built, as shown in Fig. 8, where a single-mode bare fiber $(125 \mu \mathrm{m}$ in diameter) is fixed at one end. The jacket and buffer layers are stripped before attachment to reduce the stiffness of the fiber. Photoresist or other glue is pre-applied on the contact point of the fiber and then the MEMS stage is slowly moved using a micro-positioner to the fiber to form a firm connection between the fiber and the micro-platform. A convex lens $(\mathrm{f}=6 \mathrm{~cm})$ is placed after the fiber tip to focus the light beam coming out of the fiber tip. To measure the tip deflection, a position sensitive detector (PSD) is used to record the positions of the moving light spot. The fiber tip is placed at a distance $d_{1}$ ( $\left.\mathrm{f}<\mathrm{d}_{1}<2 \mathrm{f}\right)$ and the PSD is placed at the distance $\mathrm{d}_{2}\left(\mathrm{~d}_{2}>2 \mathrm{f}\right)$ away from the convex lens. The actual displacement is calculated based on the range measured by PSD divided by the ratio $d_{2} / d_{1}$. Just for the purpose of evaluating the capability of this fiber scanner, the total length of the fiber tip was conveniently chosen as $45 \mathrm{~mm}$ and different fixing points $(20 \mathrm{~mm}, 35 \mathrm{~mm})$ were investigated in the experiments. DC voltages are applied to the LVD actuators which means they are working at non-resonance mode.

Fig. 9 shows experiment results of the tip deflection of the $45 \mathrm{~mm}$ long fiber with different fixed end length ratio: $20 \mathrm{~mm}(a=0.45)$ and $35 \mathrm{~mm}(a=0.78)$, with the calculated results based on Eq. (6) as a contrast. The maximum scanning range is $1.65 \mathrm{~mm}$ for the fixed end length of $20 \mathrm{~mm}$, and 0.92 $\mathrm{mm}$ for $35 \mathrm{~mm}$. The experiment results agree with the calculation results well.

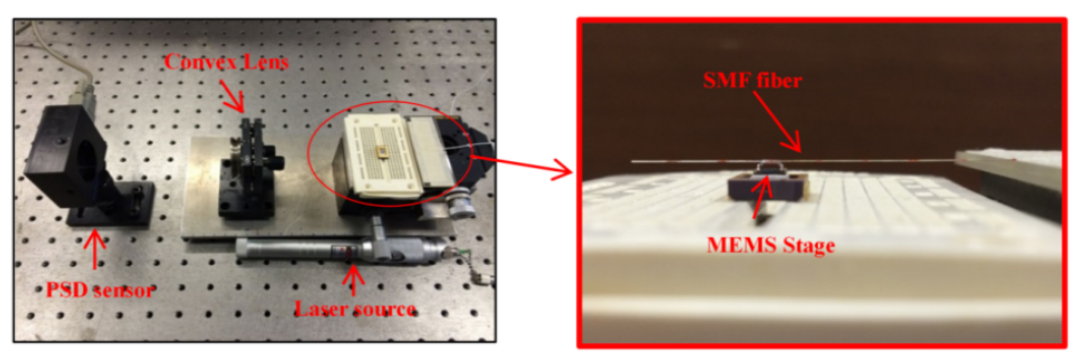

Fig. 8 Free-space experiment setup. 


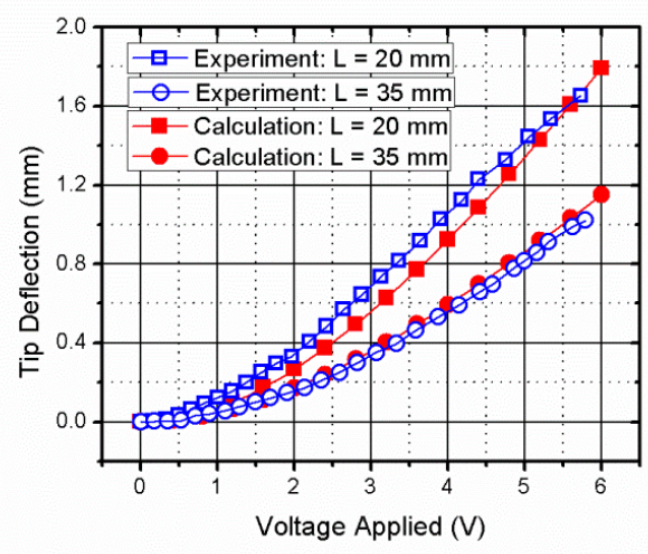

Fig. 9 Tip deflection versus applied DC voltage: Experiment and modeling results for fixed end length $\mathrm{L}=20$ $\mathrm{mm}$ and $35 \mathrm{~mm}$.

The frequency response of the fiber-stage scanning system is measured using a PSD. By recording the amplitudes of the tip deflections at different frequencies, the frequency response was achieved. The two sets of LVD actuators on both sides of the micro-platform are actuated by the same voltage signal simultaneously. Fig. 10(a) shows the frequency response of the tip deflection in the out-of-plane direction, where the first resonance peak is $107 \mathrm{~Hz}$ and the Q factor is around 20. In this case, the total length of fiber is $40 \mathrm{~mm}$, the fixed end length is $25 \mathrm{~mm}$, and the free end length is 15 $\mathrm{mm}$, respectively. Fig. 10(b) shows the COMSOL simulation result with the same lengths setting, the first mode resonant frequency is $111.6 \mathrm{~Hz}$, meanwhile, the calculated result using Eq. (16) is $101.1 \mathrm{~Hz}$, both the simulation and calculation results agree with the experiment result well with error less than $10 \%$.

Fig. 11(a) shows a non-resonant scan pattern at a driving voltage $(2.5+2.5 \sin \omega \mathrm{t}) \mathrm{V}$; the scan pattern was captured with a $5 \mathrm{~s}$ exposure time since the scan frequency was only $1 \mathrm{~Hz}$. The actual scan range was $1.3 \mathrm{~mm}$, measured using a PSD sensor. The corresponding power consumption was varying from 0 to $203 \mathrm{~mW}$. Fig. 11(b) shows a resonant scan pattern at a voltage of $(3+0.75 \sin \omega \mathrm{t}) \mathrm{V}$ with a frequency of $108 \mathrm{~Hz}$. This resonance is smaller than the resonance frequency of either the MEMS stage $(\mathrm{f}=150 \mathrm{~Hz})$ or the free end of the fiber $(\mathrm{f}=438 \mathrm{~Hz} @ \mathrm{~L}=15 \mathrm{~mm})$. The latter two resonance frequencies are from measurement shown in Fig. 7(b) and calculation according to the fiber length. The actual resonant scan range shown is about $0.9 \mathrm{~mm}$ and the power consumption was varying from 23 to $177 \mathrm{~mW}$.

Regarding the temperature change across the fiber during actuation, as mentioned in section 3.1, about $7 \%$ of the thermal power will flow into the micro-platform and the fiber through the $\mathrm{SiO}_{2}$ isolation bridge. Assuming the photoresist between the fiber and the micro-platform is $20 \mu \mathrm{m}$ thick and the contact area is $300 \mu \mathrm{m} \times 125 \mu \mathrm{m}$, the thermal resistance of the photoresist layer is about 2600 $\mathrm{K} / \mathrm{W}$. For the fiber tip, the main heat path is from the fiber-stage contact point to the fixed base. If the fixed end is $25 \mathrm{~mm}$, the thermal resistance of the fiber, $R_{T \text { f }}$, is about $1.46 \times 10^{6} \mathrm{~K} / \mathrm{W}$. The thermal resistance of the convection loss from the surface of the micro-platform, $R_{T p}$, which is in parallel with $\mathrm{R}_{\mathrm{T} \text { f }}$, is about $660 \mathrm{~K} / \mathrm{W}$ and much smaller than $\mathrm{R}_{\mathrm{Tf}}$. So the maximum temperature change of the fiber at the fiber-stage contact point is $\Delta T_{\text {fiber }} \approx 7 \% \times \frac{R_{T p}}{R_{T f+R_{T p}}} \times P_{E, \max } \times R_{T f}=28 \mathrm{~K}$. So the average 
temperature change of the fiber is about $14 \mathrm{~K}$. As the thermal expansion coefficient of the fiber is about $5.5 \times 10^{-7} \mathrm{~K}^{-1}$, the total length change of the $40 \mathrm{~mm}$ fiber is less than $0.3 \mu \mathrm{m}$, which is much smaller than the coherent length of a broadband light source (typically $5 \mu \mathrm{m}$ ) for OCT and thus can be ignored. In additional, as the thermal capacitance of the $25 \mathrm{~mm}$ fix-end fiber is about $1 \mathrm{~mJ} / \mathrm{K}$, so its thermal time constant is greater than 500s. That means the temperature variation on the fiber will be much smaller than $14 \mathrm{~K}$ even when the thermal actuation $\mathrm{AC}$ voltage signal has a frequency as low as $0.01 \mathrm{~Hz}$.

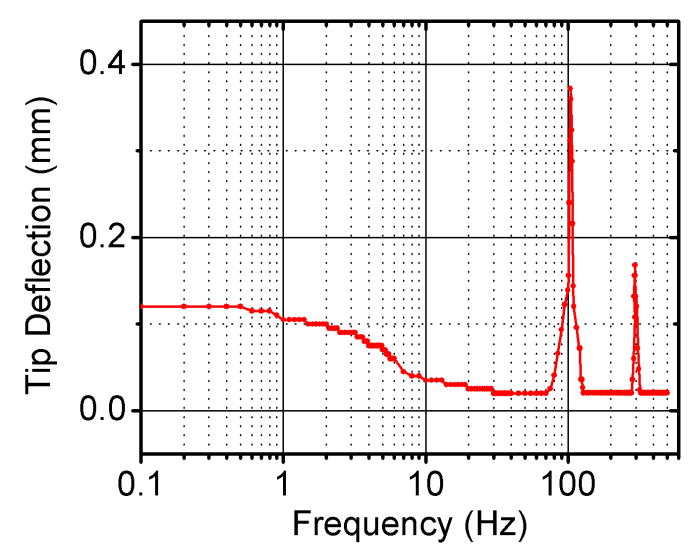

(a)

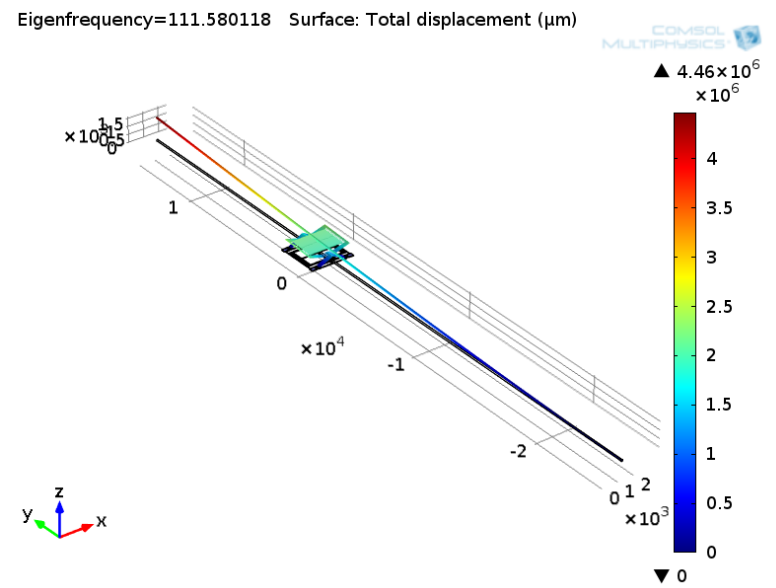

(b)

Fig. 10 (a) Experiment result of frequency response of the fiber-stage scanning system with a voltage signal of $(3+0.2 \sin \omega \mathrm{t}) \mathrm{V}$. (b) COMSOL simulation result for the fundamental resonance. 



Fig. 11 Photos of the scanning and scan patterns of the fiber-stage scanning system (the free end length $\mathrm{L}=15 \mathrm{~mm}$ and the fixed length $\mathrm{L}=25 \mathrm{~mm}$ ). (a) Non-resonant scan. (b) Resonant scan.

\section{Conclusion}

A method of using an electrothermal MEMS stage for fiber scanning has been demonstrated. The electrothermal MEMS stage with LVD bimorph actuators shows its ability to move a fiber tip to a millimeter range with small input voltage. Analytical models for both static response and the fundamental resonance of the MEMS-fiber assembly have been developed and verified experimentally. The advantages of large actuation force and large displacement of the electrothermal MEMS stage make it suitable for forward-viewing, large range scanning systems. This MEMS-fiber scanning system has large potential for probe miniaturization which can be used in many different fiber-optical applications. In addition, in-plane actuators also can be integrated with electrothermal LVD bimorph actuators based on MEMS technology and thus accomplish 3D scanning.

\section{Acknowledgments}

This work was supported by the National Science Foundation under the award \#1002209 and the National Institutes of Health R01 CA153023. Device fabrication was done in the Nanoscale Research Facility of the University of Florida.

\section{References}

[1] A. D. Mehta, J. C. Jung, B. A. Flusberg, and M. J. Schnitzer, Fiber optic in vivo imaging in the mammalian nervous system, Curr. Opin. Neurobiol. 14 (5) (2004) 617-628.

[2] B. A. Flusberg, E. D. Coker, W. Piyawattanametha, J. C. Jung, E. L. Cheung, M.J. Schnitzer, Fiber-optic fluorescence imaging, Nat. Methods 2 (12) (2005) 941-950.

[3] T. Xie, D. Mukai, S. Guo, M. Brenner, Z. Chen, Fiber optic bundle based optical tomography, Opt. Lett. 30 (14) (2005) 1803-1805.

[4] P. H. Tran, D. Mukai, M. Brenner, Z. Chen, In vivo endoscopic optical coherence tomography by use of a rotational microelectromechanical system probe, Opt. Lett, 29 (11) (2004) 1236-1238.

[5] J. J. Sun, S. G. Guo, L. Wu, L. Liu, S.W. Choe, B. S. Sorg, and H. Xie, 3D in vivo optical coherence 
tomography based on a low-voltage, large-scan-range 2D MEMS mirror, Opt. Express 18 (12) (2010) 1206512075 .

[6] X. M. Liu, M. J. Cobb, Y. C. Chen, M. B. Kimmey, and X. D. Li, Rapid-scanning forward-imaging miniature endoscope for real-time optical coherence tomography, Opt. Lett. 29 (15) (2004) 1763-1765.

[7] D. R. Rivera, C. M. Brown, D. G. Ouzounov, I. Pavlova, D. Kobat, W. W. Webb, and C. Xu. Compact and flexible raster scanning multiphoton endoscope capable of imaging unstained tissue, Proc. Natl Acad. Sci. USA 108 (43) (2011) 17598-17603.

[8] N.R. Munce, A. Mariampillai, B. A. Standish, M. Pop, K. J. Anderson, G. Y. Liu, T. Luk, B. K. Courtney, G. A. Wright, I. A. Vitkin and X. D. Yang, Electrostatic forward-viewing scanning probe for Doppler optical coherence tomography using a dissipative polymer catheter, Optics Letters 33 (7) (2008) 657-659.

[9] B. H. Hendriks, W. C. Bierhoff, J. J. Horikx, A. E. Desjardins, C. A. Hezemans, G. W. Hooft, G. W. Lucassen, N. Mihajlovic, High-resolution resonant and nonresonant fiber scanning confocal microscope, J Biomed. Opt. 16 (2) (2011) 026007-026007

[10] $\mathrm{L}$. Wu and $\mathrm{H}$. Xie, A large vertical displacement electrothermal bimorph microactuator with very small lateral shift, Sens. Actuators A 145-146 (2008) 371-379..

[11] K. Jia, S. Pal, H. Xie, An electrothermal tip-tilt-piston micromirror based on folded dual S-shaped bimorphs, J. Microelectromech. Syst. 18 (5) (2009) 1004-1015.

[12] D. J. Bell, T. J. Lu, N. A. Fleck and S. M. Spearing, MEMS actuators and sensors: observations on their performance and selection for purpose, J. Micromech. Microeng., 15 (7) (2005) 153-164.

[13] J. Wei, T. Chu Duc, G. K. Lau, P. M. Sarro, Novel electrothermal bimorph actuator for large out-of-plane displacement and force, IEEE MEMS, Tucson, AZ, USA, Jan. 13-17, 2008, pp. 46-49.

[14] S. Pal, H. Xie. A parametric dynamic compact thermal model of an electrothermally actuated micromirror. J. Micromech. Microeng. 19 (6) (2009) 065007-065016.

[15] W.H. Chu, M. Mehregany, R. L. Mullen, Analysis of tip deflection and force of a bimetallic cantilever microactuator, J. Micromech. Microeng. 6 (3) (1993) 4-7.

[16] S. Senturia, Microsystem design, Kluwer, Boston, MA, 2001, pp. 213-218.

[17] L. Wu, Low-voltage, large-range MEMS optical scanner and their applications. Ph.D Thesis, University of Florida, 2009, pp. 73-74.

[18] G. Lammel, S. Schweizer, P. Renaud, Optical microscanners and microspectrometers using thermal bimorph actuators, Kluwer Academic Publishers, Dordrecht, 2002.

[19] S. T. Todd, H. Xie, An electrothermomechanical lumped element model of an electrothermal bimorph actuator, J. Microelectromech. Syst, 17 (1) (2008) 213-225.

[20] S. Pal, H. Xie. Distributed and lumped element models for a bimorph-actuated micromirror, J. Micromech. Microeng. 20(4) (2010) 045020.

[21] C. Y. Wang, Minimum Stiffness of an Internal Elastic Support to Maximize the Fundamental Frequency of a Vibrating Beam, J. Sound Vib. 259 (1) (2003) 229-232.

[22] D. Wang, M. I. Friswell, Y. Lei, Maximizing the natural frequency of a beam with an intermediate elastic support, J. Sound Vib. 291 (3) (2006) 1229-1238.

[23] J. M. Liu, Temperature dependence of elastic stiffness in Aluminum alloys measured with non-contact electromagnetic acoustic transducers (EMATS), IEEE Ultrasonics Symposium, 1984, pp. 972-974.

[24] S. Pal, H. Xie. Fabrication of robust electrothermal MEMS devices using aluminum-tungsten bimorphs and polymide thermal isolation. J. Micromech. Microeng. 22 (11) (2012) 115036-115049. 